\title{
A class of total variation minimization problems on the whole space
}

\author{
M. Agueh* \& G. Carlier ${ }^{\dagger}$
}

December 12, 2007

\begin{abstract}
Motivated by the sharp $L^{1}$ Gagliardo-Nirenberg inequality, we prove by elementary arguments that given two increasing functions $F$ and $G$, solving the variational problem

$$
\inf \left\{E_{ \pm}(u)=\int_{\mathbb{R}^{n}} \mathrm{~d}|\nabla u| \pm \int_{\mathbb{R}^{n}} F(|u|): \int_{\mathbb{R}^{n}} G(|u|)=1\right\}
$$

amounts to solve a one-dimensional optimization problem. Under appropriate conditions on the nonlinearities $F$ and $G$, the infimum is attained and the minimizers are multiple of characteristic functions of balls. Several variants and applications are discussed, among which some sharp inequalities and nonexistence and existence results to some PDEs involving the 1-Laplacian.
\end{abstract}

Keywords: Total variation, rearrangements, sharp inequalities, 1-Laplacian.

AMS Subject Classification 2000: 49J40, 39B62, 35J20.

\section{Introduction}

In what follows, we will assume that $n$ is a given integer with $n \geq 2,|$.$| will denote both$ the euclidean norm of $\mathbb{R}^{n}$ and the absolute value function, $B_{\rho}$ the open ball with radius $\rho$ and center 0 for $||,. \mathcal{L}^{n}$ the $n$-dimensional Lebesgue measure and $\gamma_{n}:=\mathcal{L}^{n}\left(B_{1}\right)$. If $u$ : $\mathbb{R}^{n} \rightarrow \mathbb{R}$ is a measurable function, its total variation is defined by:

$$
\|\nabla u\|_{\mathcal{M}\left(\mathbb{R}^{n}\right)}:=\sup \left\{\int_{\mathbb{R}^{n}} u \operatorname{div} \Phi: \Phi \in C_{c}^{1}\left(\mathbb{R}^{n}, \mathbb{R}^{n}\right),|\Phi(x)| \leq 1 \forall x \in \mathbb{R}^{n}\right\} .
$$

*Department of Mathematics and Statistics, University of Victoria, PO. Box. 3045 STN CSC, Victoria B.C., Canada, V8W 3P4, agueh@math.uvic.ca. This author is supported by a grant from the Natural Science and Engineering Research Council of Canada.

${ }^{\dagger}$ CEREMADE, UMR CNRS 7534, Université Paris Dauphine, PL. de Lattre de Tassigny, 75775 Paris Cedex 16, France, carlier@ceremade.dauphine.fr 
When $\|\nabla u\|_{\mathcal{M}\left(\mathbb{R}^{n}\right)}<\infty, u$ will be said to be of finite total variation, and we will often write

$$
\|\nabla u\|_{\mathcal{M}\left(\mathbb{R}^{n}\right)}=\int_{\mathbb{R}^{n}} \mathrm{~d}|\nabla u|
$$

to emphasize the fact that $\nabla u$ is a vector-measure. Note that if $\nabla u \in L^{1}\left(\mathbb{R}^{n}\right)$, then the total variation of $u$ is simply $\int_{\mathbb{R}^{n}}|\nabla u|=\|\nabla u\|_{L^{1}\left(\mathbb{R}^{n}\right)}$. For $q \in[1, \infty]$, we define

$$
D^{1, q}\left(\mathbb{R}^{n}\right):=\left\{u \in L^{q}\left(\mathbb{R}^{n}\right):\|\nabla u\|_{\mathcal{M}\left(\mathbb{R}^{n}\right)}<\infty\right\}
$$

and $\operatorname{BV}\left(\mathbb{R}^{n}\right)=D^{1,1}\left(\mathbb{R}^{n}\right)$. We also define $1^{*}:=n /(n-1)$.

Given two increasing functions $F$ and $G$ on $[0, \infty)$ such that $F(0)=G(0)=0$ and $\lim _{\infty} G=\infty$, we use a symmetrization argument and a one-dimensional optimization to find a condition which ensures existence of a minimizer to the variational problem

$$
m_{ \pm}:=\inf \left\{\int_{\mathbb{R}^{n}} \mathrm{~d}|\nabla u| \pm \int_{\mathbb{R}^{n}} F(|u|): \int_{\mathbb{R}^{n}} G(|u|)=1\right\} .
$$

Under this condition, we determine explicitly the value $m_{ \pm}$and all the minimizers $u$ of this problem (they are all characteristic functions of balls). This is the main result of this paper which is presented in theorem 2.1 of the next section. In section 3 , we extend this theorem to arbitrary norms $\|$.$\| of \mathbb{R}^{n}$ and to more general functions $F$ which are difference of two increasing functions taking the value 0 at the origin. Several applications are discussed in section 4, among which some sharp inequalities and applications involving 1-Laplacian type PDEs. For example we show in section 4.1 that if we take $F(t)=t \ln t$ and $G(t)=t$, the main theorem yields the following sharp " $L^{1}$ logarithmic-Sobolev type" inequality:

$$
\int_{\mathbb{R}^{n}}|u| \ln \left(e^{n} \gamma_{n}|u|\right) \leq \int_{\mathbb{R}^{n}} \mathrm{~d}|\nabla u|+\|u\|_{L^{1}\left(\mathbb{R}^{n}\right)} \ln \left(\|u\|_{L^{1}\left(\mathbb{R}^{n}\right)}\right), \quad \forall u \in \mathrm{BV}\left(\mathbb{R}^{n}\right)
$$

which gives a quantitative form of the fact that $u$ is in the Orlicz space $L \ln L$ provided that $u$ is in $\operatorname{BV}\left(\mathbb{R}^{n}\right)$.

Our main results may be viewed as a generalization of the sharp $L^{1}$ GagliardoNirenberg inequality that we now recall. First, the $L^{1}$ Sobolev inequality reads as

$$
\|u\|_{L^{1^{*}\left(\mathbb{R}^{n}\right)}} \leq \frac{1}{n \gamma_{n}^{1 / n}}\|\nabla u\|_{\mathcal{M}\left(\mathbb{R}^{n}\right)} \quad \forall u \in \mathrm{BV}\left(\mathbb{R}^{n}\right) .
$$

Invoking the isoperimetric inequality, $\left(n \gamma_{n}^{1 / n}\right)^{-1}$ is the best constant and the only extremal functions are characteristic functions of balls. Now, let $q$ and $s$ be such that $1 \leq q<s<1^{*}$ and write

$$
\frac{1}{s}=\frac{(1-\theta)}{q}+\frac{\theta}{1^{*}} \text { i.e. } \theta=\frac{n(s-q)}{s(n-q(n-1))} .
$$

Recalling the interpolation inequality

$$
\|u\|_{L^{s}\left(\mathbb{R}^{n}\right)} \leq\|u\|_{L^{q}\left(\mathbb{R}^{n}\right)}^{1-\theta}\|u\|_{L^{1^{*}\left(\mathbb{R}^{n}\right)}}^{\theta},
$$


we deduce from (1) and (2) the $L^{1}$ Gagliardo-Nirenberg inequality

$$
\|u\|_{L^{s}\left(\mathbb{R}^{n}\right)} \leq\left(\frac{1}{n \gamma_{n}^{1 / n}}\right)^{\theta}\|\nabla u\|_{\mathcal{M}\left(\mathbb{R}^{n}\right)}^{\theta}\|u\|_{L^{q}\left(\mathbb{R}^{n}\right)}^{1-\theta}, \forall u \in D^{1, q}\left(\mathbb{R}^{n}\right) .
$$

Now we remark that characteristic functions of balls are also extremals in the interpolation inequality (2). This proves that $\left(n \gamma_{n}^{1 / n}\right)^{-\theta}$ is the best constant in the $L^{1}$ GagliardoNirenberg inequality (3) and that characteristic functions of balls are extremals in (3) (and they are the only ones again by standard isoperimetry). By a scaling argument, the optimal constant in (3) is related to the variational problem $m_{+}$with $F(t)=t^{q} / q$ and $G(t)=t^{s}$. The aim of the present paper is then precisely to generalize these well-known facts (extremality of characteristic of balls and expression of the best constant) to more general variational problems involving the total variation.

We end this introduction by remarking that in the special case where $F(t)=t$ and $G(t)=t^{s}$ with $s<1^{*}$ (i.e. the special case $q=1$ in the Gagliardo-Nirenberg inequality (3)), one may recover our main result by optimal transportation arguments using a duality method as in $[5,3]$. This is not surprising because the sharp $L^{p}$ GagliardoNirenberg inequalities for $p>1$ are obtained via optimal transportation (see [5]) when $q=1+s(p-1) / p$ (and $q=p(s-1) /(p-1)$ ), and $q=1$ is the limit case as $p \rightarrow 1$; for more discussions on this topic, we refer to $[1,2]$. Note that for $p>1$, these inequalities are first derived in their sharp form by Del Pino and Dolbeault [6] . Let us recall that, as shown in the previous paragraph, all the sharp $L^{1}$ Gagliardo-Nirenberg inequalities can be derived directly from the $L^{1}$ Sobolev inequality via the interpolation inequality, and there exists a proof of the sharp $L^{1}$ Sobolev inequality (equivalently the isoperimetric inequality) via optimal transportation $[10,5]$. But we emphasize that the optimal transportation approach does not require the isoperimetric inequality, contrarily to the proof presented in this work.

\section{Main result}

We consider the following variational problems:

$$
m_{+}:=\inf \left\{E_{+}(u)=\int_{\mathbb{R}^{n}} \mathrm{~d}|\nabla u|+\int_{\mathbb{R}^{n}} F(|u|): \int_{\mathbb{R}^{n}} G(|u|)=1\right\}
$$

and

$$
m_{-}:=\inf \left\{E_{-}(u)=\int_{\mathbb{R}^{n}} \mathrm{~d}|\nabla u|-\int_{\mathbb{R}^{n}} F(|u|): \int_{\mathbb{R}^{n}} G(|u|)=1\right\} .
$$

In both variational problems above, it is intended that the minimization is performed over functions $u$ having finite total variation and satisfying the constraint $\int_{\mathbb{R}^{n}} G(|u|)=1$.

Throughout this section, we will assume that $F$ and $G$ are two continuous, strictly increasing functions $[0, \infty) \rightarrow[0, \infty)$ such that $F(0)=G(0)=0$, and that $F$ and $G$ are $C^{1}$ on $(0, \infty)$ and $\lim _{\infty} G=\infty$. The assumptions on $G$ imply that if $u$ is such that 
$G(|u|) \in L^{1}$ then $u$ vanishes at infinity in the sense that $\{|u|>t\}$ has finite measure for every $t>0$ (more precisely $G(t) \mathcal{L}^{n}(\{|u|>t\}) \leq 1$ so that $\lim _{t \rightarrow \infty} \mathcal{L}^{n}(\{|u|>t\})=0$ ). For such a function $u$, one can then define the Schwarz symmetrization or radiallysymmetric decreasing rearrangement of $u, u^{\star}$, by:

$$
u^{\star}(x):=\sup \left\{t \geq 0: \mathcal{L}^{n}(\{|u|>t\})>\gamma_{n}|x|^{n}\right\},
$$

i.e., $u^{\star}$ is the only radially-symmetric decreasing function whose level sets have the same measure as those of $|u|$. By equimeasurability of rearrangements, we have

$$
\int_{\mathbb{R}^{n}} F(|u|)=\int_{\mathbb{R}^{n}} F\left(\left|u^{\star}\right|\right), \int_{\mathbb{R}^{n}} G(|u|)=\int_{\mathbb{R}^{n}} G\left(\left|u^{\star}\right|\right),
$$

and by Pòlya-Szegö principle, we have

$$
\int_{\mathbb{R}^{n}} \mathrm{~d}\left|\nabla u^{\star}\right| \leq \int_{\mathbb{R}^{n}} \mathrm{~d}|\nabla(|u|)| \leq \int_{\mathbb{R}^{n}} \mathrm{~d}|\nabla u| .
$$

This yields $E_{ \pm}\left(u^{\star}\right) \leq E_{ \pm}(u)$ provided $u$ has finite total variation and $u^{*}$ is admissible for (4)-(5) when $u$ is. Our main result is given by the following

Theorem 2.1. Defining $m_{+}$and $m_{-}$respectively by (4) and (5), one has:

$$
m_{ \pm}=\inf _{\alpha>0} H_{ \pm}(\alpha)
$$

with

$$
H_{ \pm}(\alpha):=n \gamma_{n}^{1 / n} \frac{\alpha}{G(\alpha)^{(n-1) / n}} \pm \frac{F(\alpha)}{G(\alpha)} .
$$

Moreover, if the set of minimizers of (6),

$$
V_{ \pm}:=\left\{\alpha>0, H_{ \pm}(\alpha)=m_{ \pm}\right\}
$$

is nonempty, then the set of minimizers of $E_{ \pm}$under the constraint $\int_{\mathbb{R}^{n}} G(|u|)=1$ is nonempty as well, and is explicitly given by

$$
\left\{u_{\alpha}\left(x_{0}+.\right), x_{0} \in \mathbb{R}^{n}, \alpha \in V\right\} \cup\left\{-u_{\alpha}\left(x_{0}+.\right), x_{0} \in \mathbb{R}^{n}, \alpha \in V\right\}
$$

where

$$
u_{\alpha}:=\alpha \chi_{B_{\rho_{\alpha}}} \text { and } \rho_{\alpha}:=\frac{1}{\gamma_{n}^{1 / n} G(\alpha)^{1 / n}}
$$

Proof. By the rearrangement arguments recalled above, we see that up to replacing $u$ by $u^{\star}$, we can restrict the variational problems (4) and (5) to nonnegative, radiallysymmetric, l.s.c., nonincreasing functions, $u$, with finite total variation such that $\int_{\mathbb{R}^{n}} G(u)=$ 1. For such a function $u$, there is a nonincreasing function $\beta: \mathbb{R}_{+} \rightarrow \mathbb{R}_{+}$such that for a.e. $t \geq 0$, one has

$$
A_{t}:=\{u>t\}=B_{\beta(t)} .
$$


Set

$$
v:=\beta^{n}
$$

and recall that $n \gamma_{n}$ is the $(n-1)$-dimensional surface area of the unit sphere. Using the Fleming-Rishel ([8]) co-area formula and Cavalieri formula, and denoting by $P(A)$ the perimeter of a ball $A \subset \mathbb{R}^{n}$, one can write:

$$
\begin{aligned}
E_{ \pm}(u) & =\int_{0}^{\infty} P\left(A_{t}\right) d t \pm \int_{0}^{\infty} \mathcal{L}^{n}(\{F(u)>t\}) d t \\
& =n \gamma_{n} \int_{0}^{\infty} \beta^{n-1}(t) d t \pm \gamma_{n} \int_{0}^{F(\infty)} \beta^{n}\left(F^{-1}(t)\right) d t \\
& =n \gamma_{n} \int_{0}^{\infty} v^{(n-1) / n}(t) d t \pm \gamma_{n} \int_{0}^{\infty} F^{\prime}(t) v(t) d t \\
& =: J_{ \pm}(v) .
\end{aligned}
$$

Similarly, we have:

$$
\int_{\mathbb{R}^{n}} G(u)=\gamma_{n} \int_{0}^{\infty} G^{\prime}(t) v(t) d t
$$

Then, the variational problems (4)-(5) transform into the optimization problem:

$$
m_{ \pm}=\inf \left\{J_{ \pm}(v): v \in K\right\}
$$

where $K$ denotes the set of nonnegative nonincreasing functions $v:(0, \infty) \rightarrow(0, \infty)$ that satisfy the linear constraint

$$
\int_{0}^{\infty} G^{\prime}(t) v(t) d t=1 / \gamma_{n}
$$

We shall now show that the minimizer of (11), if it exists, is attained at some function $v$ of the form $\frac{\chi_{[0, \alpha]}}{\gamma_{n} G(\alpha)}$. For every $\alpha>0$, define then

$$
w_{\alpha}:=\frac{\chi_{[0, \alpha]}}{\gamma_{n} G(\alpha)} .
$$

One has $w_{\alpha} \in K$, and by construction $J_{ \pm}\left(w_{\alpha}\right)=H_{ \pm}(\alpha)$. This obviously yields

$$
m_{ \pm} \leq \inf _{\alpha>0} J_{ \pm}\left(v_{\alpha}\right)=\inf _{\alpha>0} H_{ \pm}(\alpha) .
$$

To prove the converse inequality, we first remark that any $v \in K$ can be represented as

$$
v(t)=\int_{0}^{\infty} w_{\alpha}(t) d \mu_{v}(\alpha) \text { for a.e. } t>0,
$$

for some probability measure $\mu_{v}$ on $[0, \infty)$. Indeed, if $v \in K$, one can write $v(t)=$ $\theta_{v}([t, \infty))$ for a.e. $t$, where $\theta_{v}$ is a nonnegative measure on $[0, \infty)$. Setting $d \mu_{v}(\alpha)=$ $\gamma_{n} G(\alpha) d \theta_{v}(\alpha)$, we see that

$$
v(t)=\int_{t}^{\infty} d \theta_{v}(\alpha)=\int_{t}^{\infty} \frac{d \mu_{v}(\alpha)}{\gamma_{n} G(\alpha)}=\int_{0}^{\infty} w_{\alpha}(t) d \mu_{v}(\alpha) .
$$


To see that $\mu_{v}$ is a probability measure, we use Fubini's Theorem, (12), and the fact that $G(0)=0$ to have:

$$
\begin{array}{r}
\int_{0}^{\infty} d \mu_{v}(\alpha)=\gamma_{n} \int_{0}^{\infty} G(\alpha) d \theta_{v}(\alpha)=\gamma_{n} \int_{0}^{\infty}\left(\int_{0}^{\alpha} G^{\prime}(t) d t\right) d \theta_{v}(\alpha) \\
=\gamma_{n} \int_{0}^{\infty} G^{\prime}(t)\left(\int_{t}^{\infty} d \theta_{v}(\alpha)\right) d t=\gamma_{n} \int_{0}^{\infty} G^{\prime}(t) v(t) d t=1
\end{array}
$$

Now, consider an arbitrary $v$ in $K$, and write it in the form (13) for some probability measure $\mu_{v}$ on $[0, \infty)$. Since $J_{ \pm}$is concave, Jensen's inequality gives that

$$
J_{ \pm}(v)=J_{ \pm}\left(\int_{0}^{\infty} w_{\alpha} d \mu_{v}(\alpha)\right) \geq \int_{0}^{\infty} J_{ \pm}\left(w_{\alpha}\right) d \mu_{v}(\alpha)=\int_{0}^{\infty} H_{ \pm}(\alpha) d \mu_{v}(\alpha) \geq \inf _{\alpha>0} H_{ \pm}(\alpha) .
$$

Then

$$
m_{ \pm}=\inf _{v \in K} J_{ \pm}(v) \geq \inf _{\alpha>0} H_{ \pm}(\alpha)
$$

which proves (6).

Next, assume that $V_{ \pm} \neq \emptyset$, let $\alpha \in V_{ \pm}$and $u_{\alpha}$ be defined by (8). Then the function $v \in K$ defined by (9)-(10) that is associated to $u_{\alpha}$ is $w_{\alpha}$, and by a direct computation $E_{ \pm}\left(u_{\alpha}\right)=J_{ \pm}\left(w_{\alpha}\right)=H_{ \pm}(\alpha)=m_{ \pm}$so that $u_{\alpha}$ is optimal in (4)-(5).

Furthermore, if $\bar{u}$ is another optimal solution in (4)-(5) (hence vanishing at infinity and of finite total variation), as before define $u:=\bar{u}^{\star}, v$ by formulas (9)-(10), and write $v$ in the form (13) for some probability measure $\mu_{v}$ on $[0, \infty)$. By the concavity of $J_{ \pm}$, we have as before

$$
m_{ \pm}=J_{ \pm}\left(\int_{0}^{\infty} w_{\alpha} d \mu_{v}(\alpha)\right) \geq \int_{0}^{\infty} H_{ \pm}(\alpha) d \mu_{v}(\alpha) \geq \inf _{\alpha>0} H_{ \pm}(\alpha)=m_{ \pm}
$$

so that

$$
\int_{0}^{\infty}\left(H_{ \pm}(\alpha)-m_{ \pm}\right) d \mu_{v}(\alpha)=0
$$

And since $J_{ \pm}$is strictly concave, then $\mu_{v}$ must be the Dirac mass at some $\alpha \in V_{ \pm}$. Therefore, $v=w_{\alpha}$ and then $u=u_{\alpha}$ for some $\alpha \in V_{ \pm}$. As a consequence, $\bar{u}^{\star}=u=$ $u_{\alpha}$, which implies that $|\bar{u}|=\alpha \chi_{A}$ for some measurable set $A$ with $\mathcal{L}^{n}(A)=\mathcal{L}^{n}\left(B_{\rho_{\alpha}}\right)$. Moreover, since $|\bar{u}|$ is optimal, so is $\bar{u}^{\star}=u_{\alpha}$, and then $E_{ \pm}(|\bar{u}|)=E_{ \pm}\left(u_{\alpha}\right)$, which implies $|\bar{u}|$ and $u_{\alpha}$ have the same total variation, i.e., $P(A)=P\left(B_{\rho_{\alpha}}\right)$. Now we can invoke the isoperimetric inequality to conclude that $A$ is a translate of $B_{\rho_{\alpha}}$, i.e., $|\bar{u}|=u_{\alpha}\left(x_{0}+\right.$.) for some $x_{0} \in \mathbb{R}^{n}$. Finally, since $|\bar{u}|$ is a characteristic function and $\bar{u}$ and $|\bar{u}|$ have the same total variation, $\bar{u}$ cannot be sign changing. This proves that all minimizers of (4)-(5) are of the form $\bar{u}= \pm u_{\alpha}\left(x_{0}+\right.$.) for some $x_{0} \in \mathbb{R}^{n}$ and $\alpha \in V_{ \pm}$.

Remark 1. The previous result actually gives a necessary and sufficient condition for existence of a minimizer. If $H_{ \pm}$attains its infimum at some $\alpha \in(0,+\infty)$ then theorem 2.1 applies : there exist minimizers and they are all characteristics functions of balls. 
If the infimum of $H_{ \pm}$is not attained on $(0,+\infty)$, then there is a minimizing sequence $u_{\alpha_{n}}$ with $\alpha_{n}$ either converging to 0 (dispersion) or $\infty$ (concentration) and there is no minimizer in these cases.

\section{$3 \quad$ Variants and extensions}

\subsection{More general nonlinearities}

In the previous section, we assumed the monotonicity of $F$ (which excludes for instance the case $F(t)=t \ln (t)$ ). In fact, the proof of theorem 2.1 extends in a straightforward way to all functions $F$ of the form $F=F_{1}-F_{2}$, where $F_{1}$ and $F_{2}$ are continuous strictly increasing functions $[0, \infty) \rightarrow[0, \infty), C^{1}$ on $(0, \infty)$ and satisfy $F_{1}(0)=F_{2}(0)=0$. Indeed, for $u$ nonnegative, radially-symmetric and nonincreasing, and for $v$ defined by (9)-(10), following the proof of theorem 2.1, one has:

$$
\int_{\mathbb{R}^{n}} F(u)=\int_{\mathbb{R}^{n}}\left(F_{1}(u)-F_{2}(u)\right)=\gamma_{n} \int_{0}^{\infty}\left(F_{1}^{\prime}(t)-F_{2}^{\prime}(t)\right) v(t) d t=\gamma_{n} \int_{0}^{\infty} F^{\prime}(t) v(t) d t
$$

so that $E_{ \pm}(u)=J_{ \pm}(v)$ as before. Therefore the entire proof of theorem 2.1 carries over to the function $F=F_{1}-F_{2}$ defined as above. We will see in the next section that this extension of theorem 2.1 will enable us to treat examples of functions $F$ of the form $F(t)=t^{\beta} \ln (t)$ with $0<\beta \leq 1$.

\subsection{Other norms}

Another possible extension of theorem 2.1 is when one defines the total variation of $u$ with respect to an arbitrary norm in $\mathbb{R}^{n}$. Indeed, let \|\| be an arbitrary norm in $\mathbb{R}^{n}$, and denote by \|\|$_{*}$ its dual norm, that is,

$$
\|x\|_{*}:=\sup _{\|y\| \leq 1}(x \cdot y) .
$$

Following Alvino, Ferone, Lions and Trombetti [4], for $u$ of finite total variation on $\mathbb{R}^{n}$, we define the total variation of $u$ with respect to the norm || || as

$$
\int_{\mathbb{R}^{n}} \mathrm{~d}\|\nabla u\|:=\sup \left\{\int_{\mathbb{R}^{n}} u \operatorname{div} \Phi: \Phi \in C_{c}^{1}\left(\mathbb{R}^{n}, \mathbb{R}^{n}\right),\|\Phi(x)\|_{*} \leq 1, \forall x \in \mathbb{R}^{n}\right\} .
$$

All the ingredients needed to extend theorem 2.1 to the case of an arbitrary norm (convex symmetrization, co-area formula, Pòlya-Szegö principle and isoperimetric inequality) can be found in Alvino, Ferone, Lions and Trombetti [4]. Consider then the problem

$$
m:=\inf \left\{E(u)=\int_{\mathbb{R}^{n}} \mathrm{~d}\|\nabla u\|+\int_{\mathbb{R}^{n}} F(|u|): \int_{\mathbb{R}^{n}} G(|u|)=1\right\},
$$

with $F=F_{1}-F_{2}$, where $F_{1}, F_{2}$ and $G$ are continuous, strictly increasing functions $[0, \infty) \rightarrow[0, \infty), C^{1}$ on $(0, \infty)$ such that $F_{1}(0)=F_{2}(0)=G(0)=0$, and $\lim _{\infty} G=\infty$. 
In (14), it is intended that the minimization is performed over functions $u$ having finite total variation such that $\int_{\mathbb{R}^{n}} F_{1}(|u|)<\infty$ or $\int_{\mathbb{R}^{n}} F_{2}(|u|)<\infty$ and $\int_{\mathbb{R}^{n}} G(|u|)=1$. In the next statement, $K_{\rho}$ denotes the open ball with radius $\rho$ and center 0 for $\|\cdot\|_{*}$ and $\kappa_{n}:=\mathcal{L}^{n}\left(K_{1}\right)$. We then have the following generalization of theorem 2.1.

Theorem 3.1. Defining $m$ by (14), one has:

$$
m=\inf _{\alpha>0} H(\alpha)
$$

with

$$
H(\alpha):=n \kappa_{n}^{1 / n} \frac{\alpha}{G(\alpha)^{(n-1) / n}}+\frac{F(\alpha)}{G(\alpha)}
$$

Moreover, if the set

$$
V:=\{\alpha>0, H(\alpha)=m\}
$$

is nonempty, then the set of minimizers of $E$ under the constraint $\int_{\mathbb{R}^{n}} G(|u|)=1$ is nonempty and explicitly given by $\left\{u_{\alpha}\left(x_{0}+.\right), x_{0} \in \mathbb{R}^{n}, \alpha \in V\right\} \cup\left\{-u_{\alpha}\left(x_{0}+.\right), x_{0} \in\right.$ $\left.\mathbb{R}^{n}, \alpha \in V\right\}$ where

$$
u_{\alpha}:=\alpha \chi_{K_{\rho_{\alpha}}} \text { and } \rho_{\alpha}:=\frac{1}{\kappa_{n}^{1 / n} G(\alpha)^{1 / n}} .
$$

\section{Applications}

In this section, we apply the main theorem 3.1 to establish some sharp inequalities, and to study existence or non-existence of solutions to some partial differential equations involving the 1-Laplacian operator in $\mathbb{R}^{n}$. Throughout this section, we assume that $F$ and $G$ are as in section 3 .

\subsection{Inequalities}

Under the assumptions and notations of subsection 3.2, we easily deduce from theorem 3.1 (where we use $-F$ in place of $F$ for convenience) the following proposition:

Proposition 4.1. Assume that $F$ and $G$ are such that the problem

$$
\inf _{\alpha>0}\left\{H(\alpha):=n \kappa_{n}^{1 / n} \frac{\alpha}{G(\alpha)^{(n-1) / n}}-\frac{F(\alpha)}{G(\alpha)}\right\}
$$

has a solution $\alpha_{\infty}$, and set

$$
u_{\infty}:=\alpha_{\infty} \chi_{K_{\rho_{\infty}}} \text { where } \rho_{\infty}:=\frac{1}{\kappa_{n}^{1 / n} G\left(\alpha_{\infty}\right)^{1 / n}},
$$

and

$$
E(u):=\int_{\mathbb{R}^{n}} \mathrm{~d}\|\nabla u\|-\int_{\mathbb{R}^{n}} F(|u|) .
$$


Then the sharp inequality

$$
\int_{\mathbb{R}^{n}} F(|u|)+E\left(u_{\infty}\right) \leq \int_{\mathbb{R}^{n}} \mathrm{~d}\|\nabla u\|
$$

holds for all functions $u$ with finite total variation such that $\int_{\mathbb{R}^{n}} G(|u|)=1$. Moreover, equality occurs in (20) if and only if $u= \pm u_{\infty}\left(x_{0}+\right.$.) for some $x_{0} \in \mathbb{R}^{n}$ with $u_{\infty}$ defined by (19) for some $\alpha_{\infty}$ solving (18).

Applying proposition 4.1 with $F(t)=t \ln t$ and $G(t)=t$, we obtain the following sharp inequality which roughly states that the entropy of $u, \operatorname{Ent}_{\mathcal{L}^{n}}(u)=\int_{\mathbb{R}^{n}}|u| \ln |u|$, is bounded above by the total variation of $u$, up to some additive term $n+\ln \left(\kappa_{n}\right)$, provided that $u \in \mathrm{BV}\left(\mathbb{R}^{n}\right)$ with $\|u\|_{L^{1}\left(\mathbb{R}^{n}\right)}=1$. In this sense, this inequality can be called: sharp " $L^{1}$ logarithmic Sobolev inequality".

Corollary 4.2. For any function $u$ in $\mathrm{BV}\left(\mathbb{R}^{n}\right)$ such that $\int_{\mathbb{R}^{n}}|u|=1$, we have

$$
\int_{\mathbb{R}^{n}}|u| \ln \left(e^{n} \kappa_{n}|u|\right) \leq \int_{\mathbb{R}^{n}} \mathrm{~d}\|\nabla u\|
$$

and the optimal functions $u$ are such that $\pm \kappa_{n} u$ are characteristic functions of unit balls in $\left(\mathbb{R}^{n},\|\|_{*}\right)$.

Proof. First we observe that $F(t)=t \ln t=F_{1}(t)-F_{2}(t)$ where $F_{1}(t)=t \ln t+\sqrt{t}$ and $F_{2}(t)=\sqrt{t}$, so that $F(t)=t \ln t$ and $G(t)=t$ are admissible in proposition 4.1. Then (18) reads as

$$
\inf _{\alpha>0}\left\{H(\alpha)=n \kappa_{n}^{1 / n} \alpha^{1 / n}-\ln \alpha\right\},
$$

and it is easy to see that its unique solution is $\alpha_{\infty}=1 / \kappa_{n}$. Hence $\rho_{\infty}=\frac{1}{\kappa_{n}^{1 / n} \alpha_{\infty}^{1 / n}}=1$, $u_{\infty}=\chi_{K_{1}} / \kappa_{n}$, and by a direct computation, we have $E\left(u_{\infty}\right)=n+\ln \kappa_{n}$. Therefore, (20) reads as (21) with the optimal functions $\pm \frac{1}{\kappa_{n}} \chi_{K_{1}}\left(x_{0}+.\right), x_{0} \in \mathbb{R}^{n}$. This completes the proof.

Remark 2. It may be convenient to (equivalently) rewrite the inequality (21) of corollary 4.2 as

$$
\int_{\mathbb{R}^{n}}|u| \ln \left(e^{n} \kappa_{n}|u|\right) \leq \int_{\mathbb{R}^{n}} \mathrm{~d}\|\nabla u\|+\|u\|_{L^{1}\left(\mathbb{R}^{n}\right)} \ln \left(\|u\|_{L^{1}\left(\mathbb{R}^{n}\right)}\right), \quad \forall u \in \mathrm{BV}\left(\mathbb{R}^{n}\right)
$$

or

$$
\int_{\mathbb{R}^{n}}|u| \ln \left(\frac{e^{n} \kappa_{n}|u|}{\|u\|_{L^{1}\left(\mathbb{R}^{n}\right)}}\right) \leq \int_{\mathbb{R}^{n}} \mathrm{~d}\|\nabla u\|, \quad \forall u \in \mathrm{BV}\left(\mathbb{R}^{n}\right) .
$$


Remark 3. In fact, by chosing $F(t)=t^{\beta} \ln t$ with $0<\beta \leq 1$ and $G(t)=t$, inequality (21) generalizes to

$$
\int_{\mathbb{R}^{n}}|u|^{\beta} \ln |u|+E\left(u_{\infty}\right) \leq \int_{\mathbb{R}^{n}} \mathrm{~d}\|\nabla u\|
$$

for all $\mathrm{BV}\left(\mathbb{R}^{n}\right)$ functions $u$ satisfying $\int_{\mathbb{R}^{n}}|u|=1$, where $u_{\infty}$ is defined by (19), and $\alpha_{\infty}$ solves

$$
\inf _{\alpha>0}\left\{H(\alpha)=n \kappa_{n}^{1 / n} \alpha^{1 / n}-\alpha^{\beta-1} \ln \alpha\right\} .
$$

And the optimal functions are $u= \pm u_{\infty}\left(x_{0}+.\right), x_{0} \in \mathbb{R}^{n}$. Note that, even though it may be difficult to compute $\alpha_{\infty}$ explicitly when $\beta \neq 1$, its existence is ensured by the condition $\beta<1$.

\subsection{Link with some PDE's involving the 1-Laplacian}

For simplicity, we will restrict to the Euclidean norm of $\mathbb{R}^{n}$, though the results of this section extend naturally to arbitrary norms as in section 3.2. If $u$ is a nonnegative solution of the variational problem

$$
\inf \left\{E(u):=\int_{\mathbb{R}^{n}} \mathrm{~d}|\nabla u|+\int_{\mathbb{R}^{n}} F(|u|): \int_{\mathbb{R}^{n}} G(|u|)=1\right\},
$$

then, formally, $u$ is a nonnegative solution of the 1-Laplacian PDE

$$
-\Delta_{1} u+F^{\prime}(u)=\lambda G^{\prime}(u)
$$

where $\lambda \in \mathbb{R}$ is a Lagrange multiplier for the constraint $\int_{\mathbb{R}^{n}} G(|u|)=1$ and the 1Laplacian operator is (again formally) defined by

$$
\Delta_{1} u=\operatorname{div}\left(\frac{\nabla u}{|\nabla u|}\right) .
$$

The aim of this paragraph is to apply theorem 2.1 to derive nonexistence and existence results for some PDE's involving the 1-Laplacian. We start by some preliminaries on this operator. For $q \in[1, \infty]$ and $q^{*}$ the conjugate exponent of $q\left(\frac{1}{q}+\frac{1}{q^{*}}=1\right)$, we first define

$$
X^{q^{*}}:=\left\{\sigma \in L^{\infty}\left(\mathbb{R}^{n}, \mathbb{R}^{n}\right): \operatorname{div}(\sigma) \in L^{q^{*}}\left(\mathbb{R}^{n}, \mathbb{R}\right)\right\} .
$$

For $\sigma \in X^{q^{*}}$ and $u \in D^{1, q}\left(\mathbb{R}^{n}\right)$, (following for instance Demengel [7]), we define the distribution $\sigma \cdot \nabla u$ by

$$
\langle\sigma \cdot \nabla u, \varphi\rangle=-\int_{\mathbb{R}^{n}}(\operatorname{div} \sigma) \varphi u-\int_{\mathbb{R}^{n}}(\sigma \cdot \nabla \varphi) u, \forall \varphi \in C_{c}^{1}\left(\mathbb{R}^{n}\right) .
$$

If $\sigma \in X^{q^{*}}$ and $u \in D^{1, q}\left(\mathbb{R}^{n}\right)$, then it is easy to see that $\sigma \cdot \nabla u$ is a Radon measure on $\mathbb{R}^{n}$ which satisfies (in the sense of nonnegative measures)

$$
|\sigma \cdot \nabla u| \leq\|\sigma\|_{\infty}|\nabla u|
$$


hence in particular:

$$
\|\sigma \cdot \nabla u\|_{\mathcal{M}} \leq\|\sigma\|_{\infty} \int_{\mathbb{R}^{n}} \mathrm{~d}|\nabla u|
$$

It is also easy to check that

$$
\int_{\mathbb{R}^{n}} \sigma \cdot \nabla u=-\int_{\mathbb{R}^{n}} \operatorname{div}(\sigma) u, \forall(u, \sigma) \in D^{1, q}\left(\mathbb{R}^{n}\right) \times X^{q^{*}}
$$

and

$$
\int_{\mathbb{R}^{n}} \mathrm{~d}|\nabla u|=\sup \left\{\int_{\mathbb{R}^{n}} \operatorname{div}(\sigma) u, \sigma \in X^{q^{*}},\|\sigma\|_{\infty} \leq 1\right\} .
$$

A natural and classical way to define the 1-Laplacian is via convex analysis. More precisely, let $q \in[1,+\infty)$ and consider the following functional on $L^{q}\left(\mathbb{R}^{n}\right)$ :

$$
J_{q}(u):= \begin{cases}\int_{\mathbb{R}^{n}} \mathrm{~d}|\nabla u| & \text { if } u \in D^{1, q}\left(\mathbb{R}^{n}\right) \\ +\infty & \text { otherwise. }\end{cases}
$$

It is obvious that $J_{q}$ is a convex (and positively homogeneous) l.s.c. functional on $L^{q}\left(\mathbb{R}^{n}\right)$. For $u \in L^{q}\left(\mathbb{R}^{n}\right)$, recall that the subgradient of $J_{q}$ at $u$, denoted $\partial J_{q}(u)$, consists of all $p \in L^{q^{*}}\left(\mathbb{R}^{n}\right)$ such that

$$
J_{q}(v)-J_{q}(u) \geq \int_{\mathbb{R}^{n}} p(v-u), \forall v \in L^{q}\left(\mathbb{R}^{n}\right) .
$$

By definition, $J_{q}$ is said to be subdifferentiable at $u$ whenever $\partial J_{q}(u) \neq \emptyset$ (which in particular implies that $\left.u \in D^{1, q}\left(\mathbb{R}^{n}\right)\right)$. The following characterization of $\partial J_{q}$ is rather classical and easy to prove:

Lemma 4.3. Let $u \in L^{q}\left(\mathbb{R}^{n}\right.$ be such that $J_{q}$ is subdifferentiable at $u$. Then one has

$$
\partial J_{q}(u)=\left\{-\operatorname{div}(\sigma), \sigma \in X^{q^{*}},\|\sigma\|_{\infty} \leq 1, \sigma \cdot \nabla u=|\nabla u|\right\} .
$$

If one considers the variational problem

$$
\inf _{u \in D^{1, q}}\left\{J_{q}(u)+\int_{\mathbb{R}^{n}} f(u(x)) d x\right\}
$$

where $f$ is differentiable and satisfies

$$
|f(u)| \leq C|u|^{q},\left|f^{\prime}(u)\right| \leq C|u|^{q-1}
$$

then if $u$ solves $(24), J_{q}$ is subdifferentiable at $u$ and the following Euler-Lagrange equation is satisfied

$$
0 \in \partial J_{q}(u)+f^{\prime}(u)
$$

which can be rewritten as

$$
-\Delta_{1} u+f^{\prime}(u)=0
$$

according to the following definition: 
Definition 4.4. Let $g \in C^{0}(\mathbb{R}, \mathbb{R})$ and $q \in[1,+\infty)$. Then $u \in D^{1, q}\left(\mathbb{R}^{n}\right)$ is said to be a $D^{1, q}$ solution of the equation

$$
-\Delta_{1} u=g(u)
$$

if there exists $\sigma \in X^{q^{*}}$ such that $-\operatorname{div}(\sigma)=g(u)$ (in the sense of distributions), $\|\sigma\|_{\infty} \leq$ 1 and $\sigma \cdot \nabla u=|\nabla u|$.

Let us remark that $\sigma$ needs not be unique in the previous definition. Note also that it is required in the previous definition that $g(u) \in L^{q^{*}}\left(\mathbb{R}^{n}\right)$.

Let us now consider the variational problem (22) under the assumptions that $F$ and $G$ are strictly increasing differentiable functions on $\mathbb{R}_{+}$such that $F(0)=G(0)=0$, $G(\infty)=\infty$ and there exist $q \in(1, \infty)$ and some positive constants $A$ and $B$ such that:

$$
F(t) \leq A t^{q},\left|F^{\prime}(t)\right| \leq B t^{q-1}, \forall t \in \mathbb{R}_{+} .
$$

Theorem 4.5. If, in addition to the assumptions above, $F$ is convex and $G$ is concave on $\mathbb{R}_{+}$and $\lambda \geq 0$, then the PDE:

$$
\left\{\begin{array}{l}
-\Delta_{1} u=\lambda G^{\prime}(u)-F^{\prime}(u) \\
u \geq 0, \int_{\mathbb{R}^{n}} G(u)=1
\end{array}\right.
$$

has no $D^{1, q}$ solution.

Proof. It is easy to check that our convexity assumptions actually imply that (26) is a sufficient optimality condition for a nonnegative solution of (22). Theorem 2.1 asserts that (22) admits solutions if and only if the function

$$
H(\alpha)=n \gamma_{n}^{1 / n} \frac{\alpha}{G(\alpha)^{(n-1) / n}}+\frac{F(\alpha)}{G(\alpha)}
$$

achieves its minimum on $(0, \infty)$. Now we remark that $G^{\prime}\left(0^{+}\right)>0$ (otherwise by concavity and monotonicity $G$ would identically be 0$)$. This together with $F^{\prime}\left(0^{+}\right)=0$ implies that $H\left(0^{+}\right)=0<H(\alpha)$ for every $\alpha>0$. Therefore (22) does not possess any solution and thus neither does $(26)$. have:

The previous result applies in particular to $G(t)=t$ and $F(t)=t^{q} / q(q>1)$. We Corollary 4.6. If $q>1$, then the PDE

$$
-\Delta_{1} u=1-u^{q-1}
$$

has no $D^{1, q}$ solution.

Proof. Using $G(t)=t$ and $F(t)=t^{q} / q(q>1)$ in theorem 4.5, we have that

$$
\left\{\begin{array}{l}
-\Delta_{1} u=\lambda-u^{q-1} \\
u \geq 0, \int_{\mathbb{R}^{n}} u=1
\end{array}\right.
$$

has no $D^{1, q}$ solution. By a scaling argument, $u$ solves $(28)$ if and only $u_{\lambda}(x):=\lambda^{\frac{1}{1-q}} u\left(\frac{x}{\lambda}\right)$ solves (27). This completes the proof. 
Now, let us assume that $F=F_{1}-F_{2}$ with $F_{1}, F_{2}$ and $G$ satisfying the assumptions of subsection 3.2 and in addition $F_{1}^{\prime}(0)=F_{2}^{\prime}(0)=G^{\prime}(0)=0$ (so that $t \mapsto$ $F_{1}(|t|), F_{2}(|t|), G(|t|)$ are differentiable at 0$)$ and that there exists $\alpha_{\infty} \in(0, \infty)$ such that

$$
H\left(\alpha_{\infty}\right) \leq H(\alpha)=n \gamma_{n}^{1 / n} \frac{\alpha}{G(\alpha)^{(n-1) / n}}+\frac{F(\alpha)}{G(\alpha)}, \forall \alpha \in(0,+\infty) .
$$

We then have the following existence result:

Theorem 4.7. Under the assumptions above, the following PDE (where $\lambda \in \mathbb{R}$ is part of the unknown)

$$
\left\{\begin{array}{l}
-\Delta_{1} u=\lambda G^{\prime}(u)-F^{\prime}(u) \\
u \geq 0, \int_{\mathbb{R}^{n}} G(u)=1
\end{array}\right.
$$

admits a $\mathrm{BV}=D^{1,1}$ solution.

Proof. Thanks to theorem 3.1, the function

$$
u_{\infty}:=\alpha_{\infty} \chi_{B_{\rho_{\infty}}}, \quad \text { with } \rho_{\infty}:=\frac{1}{\gamma_{n}^{1 / n} G\left(\alpha_{\infty}\right)^{1 / n}}
$$

is a minimizer of (22). Let us now prove that $u_{\infty}$ solves (29). Indeed, define

$$
\sigma(x)= \begin{cases}-x / \rho_{\infty} & \text { if } x \in B_{\rho_{\infty}} \\ -\rho_{\infty}^{n-1} x /|x|^{n} & \text { if } x \notin B_{\rho_{\infty}}\end{cases}
$$

By construction, we have

$$
\|\sigma\|_{\infty} \leq 1, \operatorname{div} \sigma=-\frac{n}{\rho_{\infty}} \chi_{B_{\rho_{\infty}}} \text { and } \sigma \cdot \nabla u_{\infty}=\left|\nabla u_{\infty}\right|=\alpha_{\infty} \mathcal{H}^{n-1}\left\llcorner\partial B_{\rho_{\infty}}\right.
$$

(where $\mathcal{H}^{n-1}\left\llcorner\partial B_{\rho_{\infty}}\right.$ denotes the $n-1$-dimensional Hausdorff measure on $\partial B_{\rho_{\infty}}$ ). It follows that $-\operatorname{div} \sigma \in \partial J_{1}\left(u_{\infty}\right)$. Thus $u_{\infty}$ is a BV solution of (29) provided that

$$
-\operatorname{div} \sigma=\frac{n}{\rho_{\infty}} \chi_{B_{\rho_{\infty}}}=\lambda G^{\prime}\left(u_{\infty}\right)-F^{\prime}\left(u_{\infty}\right)=\left(\lambda G^{\prime}\left(\alpha_{\infty}\right)-F^{\prime}\left(\alpha_{\infty}\right)\right) \chi_{B_{\rho_{\infty}}}
$$

i.e.

$$
\frac{n}{\rho_{\infty}}=\lambda G^{\prime}\left(\alpha_{\infty}\right)-F^{\prime}\left(\alpha_{\infty}\right)
$$

If $G^{\prime}\left(\alpha_{\infty}\right) \neq 0$, then $\lambda$ is uniquely determined by solving the previous equation. Now, if $G^{\prime}\left(\alpha_{\infty}\right)=0$, using the fact that $H^{\prime}\left(\alpha_{\infty}\right)=0$, a direct computation gives

$$
0=n \gamma_{n}^{1 / n} G\left(\alpha_{\infty}\right)^{1 / n}+F^{\prime}\left(\alpha_{\infty}\right)=\frac{n}{\rho_{\infty}}+F^{\prime}\left(\alpha_{\infty}\right)
$$

and then $u_{\infty}$ solves (29) for any $\lambda \in \mathbb{R}$ in this case.

Remark 4. In the case where $F$ is increasing then it follows from (30) that $\lambda>0$. This is not surprising since in this case, by monotonicity one can replace in (22) the constraint by $\int G(|u|) \geq 1$. 
Remark 5. The previous proof actually shows that nonnegative minimizers of (22) solve the PDE (29). Obviously, for every critical point $\alpha_{\infty}$ of $H$, the corresponding function $u_{\infty}$ also solves (29). Whether there exist other solutions of this PDE (non radial for instance) is an interesting question that we do not address here.

Theorem 4.7 applies in particular to the functions $F(t)=t^{q} / q$ and $G(t)=t^{s} / s$ in the two cases $1^{*}<s<q$ and $1<q<s<1^{*}\left(1^{*}=n /(n-1)\right)$. As a consequence, we have:

Corollary 4.8. If either $1<q<s<1^{*}:=n /(n-1)$ or $1^{*}<s<q$, then the PDE

$$
-\Delta_{1} u=u^{s-1}-u^{q-1}
$$

has nontrivial nonnegative solutions in $D^{1, q}\left(\mathbb{R}^{n}\right)$.

Furthermore

$$
-\Delta_{1} u=u^{1 /(n-1)}
$$

has nontrivial nonnegative solutions in $D^{1,1^{*}}\left(\mathbb{R}^{n}\right)$.

Proof. By theorem 4.7 and remark 4, there exists $\lambda>0$ such that the PDE

$$
\left\{\begin{array}{l}
-\Delta_{1} u=\lambda u^{s-1}-u^{q-1} \\
u \geq 0, \int_{\mathbb{R}^{n}} u^{s}=1
\end{array}\right.
$$

has a solution $u$. We then use the scaling properties of the equation to show that $u_{\lambda}(x):=\lambda^{\frac{1}{s-q}} u\left(\lambda^{\frac{q-1}{s-q}} x\right)$ is a solution of $(31)$, which obviously is nonzero and nonnegative since $u$ is. If $s=1^{*}$, we choose instead $F=0$ and $G(t)=t^{1^{*}}$ to conclude the proof.

Another possible application is $F(t)=-t^{q} / q$ and $G(t)=t^{s}$ with $s<q<1+s / n$ (therefore $s<1^{*}$ ). In this case we obtain:

Corollary 4.9. If $s<q<1+s / n$ and therefore $s<1^{*}:=n /(n-1)$, then the PDE

$$
-\Delta_{1} u=u^{s-1}+u^{q-1}
$$

has nontrivial nonnegative solutions in $\mathrm{BV}\left(\mathbb{R}^{n}\right)=D^{1,1}\left(\mathbb{R}^{n}\right)$. In particular if $1<q<$ $(n+1) / n$ (that is $s=1)$, then $-\Delta_{1} u=1+u^{q-1}$ has nontrivial nonnegative solutions in $\mathrm{BV}\left(\mathbb{R}^{n}\right)$.

Proof. Since $s<q<1+s / n$, we have that $1-s / 1^{*}>q-s>0$ so that

$$
\inf _{\alpha>0}\left\{H(\alpha):=n \gamma_{n}^{1 / n} \alpha^{1-s / 1^{*}}-\alpha^{q-s} / q\right\}
$$

is attained at

$$
\alpha_{\infty}=\left[\frac{q-s}{q n \gamma^{1 / n}\left(1-s / 1^{*}\right)}\right]^{\frac{1}{1+s / n-q}} .
$$

We use theorem 4.7 and again a scaling argument to conclude the proof. 


\section{References}

[1] M. Agueh, Sharp Gagliardo-Nirenberg inequalities and Mass transport theory, Journal of Dynamics and Differential Equations, Vol. 18, No. 4, (2006), 1069-1093.

[2] M. Agueh, Sharp Gagliardo-Nirenberg inequalities via p-Laplacian type equations, Preprint 2007. Nonlinear Differential Equations Appl., (to appear).

[3] M. Agueh, N. Ghoussoub, and X. Kang. Geometric inequalities via a general comparison principle for interacting gases, Geom. Funct. Anal., 14, (2004), 215-244.

[4] A. Alvino, V. Ferone, P-L. Lions, Convex symmetrization and applications, Ann. Inst. Henri. Poincaré., 14(2) (1997), 275-293

[5] D. Cordero-Erausquin, B. Nazaret, and C. Villani. A mass transportation approach to sharp Sobolev and Gagliardo-Nirenberg inequalities, Adv. Math., 182 (2004), 307-332.

[6] M. Del-Pino, and J. Dolbeault, The optimal Euclidean $L^{p}$-Sobolev logarithmic inequaity, J. Funct. Anal. 197(1) (2003), 151-161.

[7] F. Demengel. Théorème d'existence pour des équations avec l'opérateur 1-Laplacien, première valeur propre pour $-\Delta_{1}$, C. R. Acad. Sci. Paris, I 334 (2002), 1071-1076.

[8] W. H. Fleming, and R. Rishel, An integral formula for total gradient variation, Arch. Math. (11) (1960), 218-222.

[9] R. J. McCann, A convexity theory for interacting gases and equlibrium crystals, Ph.D Thesis, Princeton University, 1994. 14, AMS, 1997.

[10] V. D. Milman, and G. Schechtman Asymptotic theory of finite-dimensional normed spaces., Springer-Verlag, Berlin, 1986. (Appendix by M. Gromov).

[11] S. T. Rachev, and L. Rüschendorf, Mass transportation problems, Vol I: Theory, SpringerVerlag, New-York, 1998.

[12] C. Villani, Topics in Optimal Transportation, Graduate Studies in Math, 58, AMS, Providence, RI, 2003. 